\title{
Leisure-Time Physical Activity and Its Association With Metabolic Risk Factors in Iranian Adults: Tehran Lipid and Glucose Study, 2005-2008
}

Bita Faam, MSc; Farhad Hosseinpanah, MD; Atieh Amouzegar, MD; Arash Ghanbarian, MD; Golaleh Asghari, MSc; Fereidoun Azizi, MD

Suggested citation for this article: Faam B, Hosseinpanah F, Amouzegar A, Ghanbarian A, Asghari G, Azizi F. LeisureTime Physical Activity and Its Association With Metabolic Risk Factors in Iranian Adults: Tehran Lipid and Glucose Study,

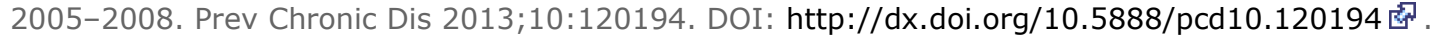

\section{Abstract}

We examined the association between leisure-time physical activity (LTPA) and metabolic syndrome (MetS) among 4,665 randomly selected adults who participated in the Tehran Lipid and Glucose Study, 2005-2008. Normal-weight participants with light LTPA had higher risk of low high-density lipoprotein cholesterol and elevated levels of triglycerides than those with vigorous LTPA. Overweight adults with moderate LTPA had higher risk of having elevated levels of fasting blood glucose than adults with vigorous LTPA and, in the same group, we found an inverse association between light LTPA and MetS after adjustment for sex, age, education levels, smoking, and calorie intake. Although participants in the normal-weight and obese groups with vigorous LTPA had higher risk of high systolic blood pressure than participants with moderate LTPA, this finding had no clinical significance. Increased LTPA is associated with decreased risk of any damaging changes in the markers of MetS.

\section{Objective}

Metabolic syndrome (MetS) is caused by a combination of an unhealthy diet, sedentary lifestyle, and genetic predisposition (1). Prevalence of MetS in South Asian populations is approximately 30\%, depending on the region, duration and extent of urbanization, and lifestyle, and in Iranian populations is $32 \%$ (2). Recommendations for prevention and treatment of MetS and its components are engaging in physical activity, losing weight, and eating a healthful diet. Leisure-time physical activity (LTPA) plays an important role in controlling MetS $(3,4)$. The aim of this study was to examine the association between LTPA and MetS among adults in the Tehran Lipid and Glucose Study (TLGS).

\section{Methods}

Initially, 9,376 adults aged 20 to 70 years were selected randomly from among TLGS participants (5) for this crosssectional study; after excluding participants who had diabetes mellitus $(\mathrm{n}=390)$, body mass index $(\mathrm{BMI})$ less than 18 $\mathrm{kg} / \mathrm{m}^{2}(\mathrm{n}=152)$, or lacked complete data for variables $(\mathrm{n}=4,169), 4,665$ remained $(1,976$ men and 2,689 women). This study was approved by the institutional review board of the Research Institute for Endocrine Sciences, Shahid Beheshti University of Medical Sciences. Demographic and biochemical variables were measured (5), and people were classified on the basis of BMI levels. We defined MetS according to the Joint Interim Statement (JIS) guidelines issued by the International Diabetes Federation Task Force on Epidemiology and Prevention; National Heart, Lung, and Blood Institute; American Heart Association; World Heart Federation; International Atherosclerosis Society; and International Association for the Study of Obesity $(6,7)$. We collected information about physical activity by using the Modifiable Activity Questionnaire (MAQ) (8) to calculate metabolic equivalent task (MET) minutes per week. High reliability (98\%) and moderate validity (47\%) were found for the MAQ translated into Persian $(8,9)$. Levels of LTPA were categorized as light (MET $<600 \mathrm{~min} / \mathrm{wk}$ ), moderate (MET 600-1,499 $\mathrm{min} / \mathrm{wk}$ ), and vigorous (MET $\geq 1,500$ $\mathrm{min} / \mathrm{wk}$ ) (10). Dietary intake was assessed by using a 168-item validated semiquantitative food frequency questionnaire (FFQ) (11). Each food and beverage was analyzed for energy (kcal) content by using the US Department of Agriculture's food composition database. Levels of education were categorized as elementary or secondary, high 
school, and bachelor's degree or higher. Participants with current or past history of smoking were categorized as smokers and those who had never smoked were categorized as nonsmokers.

\section{Statistical analysis}

We used the $\chi^{2}$ test to compare LTPA levels between BMI groups. Analysis of covariance was used to calculate the means of individual MetS components in various BMI groups. Multiple logistic regressions were performed to determine the association between individual MetS components and LTPA. All statistical analyses were performed by SPSS version 16.0 (IBM, Chicago, Illinois); tests were 2-sided, and difference was considered statistically significant at $P<.05$.

\section{Results}

Mean age of participants was 40.7 (standard deviation [SD], 13.9) years. Of these participants, 1,108 (23.7\%) were obese (BMI $\geq 30$ ). According to the JIS definition, 31.5\% of the 4,665 participants had MetS. Approximately $17 \%$ of participants had engaged in vigorous LTPA. The obese group had significantly higher weight, waist circumference, BMI, fasting blood glucose, systolic and diastolic blood pressure, and lower high-density lipoprotein (HDL) cholesterol than normal and overweight groups. The prevalence of MetS was significantly higher in the obese group (58.2\%) than among overweight (36.6\%) and normal-weight adults (6\%). We found no significant difference between BMI groups regarding LTPA categories, which were proportionally distributed between groups (Table 1).

In the obese group, systolic blood pressure and triglyceride levels differed significantly by LTPA categories $(P=.03$ for systolic blood pressure, $P=.01$ for triglyceride level) (Figure). Normal-weight adults who participated in light LTPA had a higher risk of having elevated triglycerides and reduced HDL cholesterol than did adults who participated in vigorous LTPA (odds ratio [OR] for elevated triglycerides, 1.46; 95\% confidence interval [CI], 1.01-2.14; $P=.049$ ) (OR for reduced HDL, 1.15; 95\% CI, 1.05-2.33; $P=.03$ ) (Table 2). However, these associations were attenuated after adjustment for sex, age, smoking status, education levels, and calorie intake. In the overweight group, adults who participated in moderate LTPA had higher fasting blood glucose levels than those with vigorous LTPA after adjustment for sex, age, smoking status, education levels, and calorie intake (OR, 1.65; 95\% CI, 1.37-3.23; $P=.02$ ), and the association between MetS and light LTPA was significant only after adjustment for the same variables (OR, 2.08; 95\% CI, 1.03-4.21; $P=.04$ ). Normal-weight participants with vigorous LTPA had a greater risk of having high systolic blood pressure than did those whose LTPA was moderate (OR, 0.52; 95\% CI, $0.31-0.86 ; P=.01$ ), and the same risk was found in obese people who participated in vigorous LTPA compared with light LTPA (OR, 0.60; 95\% CI, 0.41-0.91; $P$ $=.01$ ) and moderate LTPA (OR: 0.58; 95\% CI, 0.39-0.64; $P=.005$ ) (Table 2). 

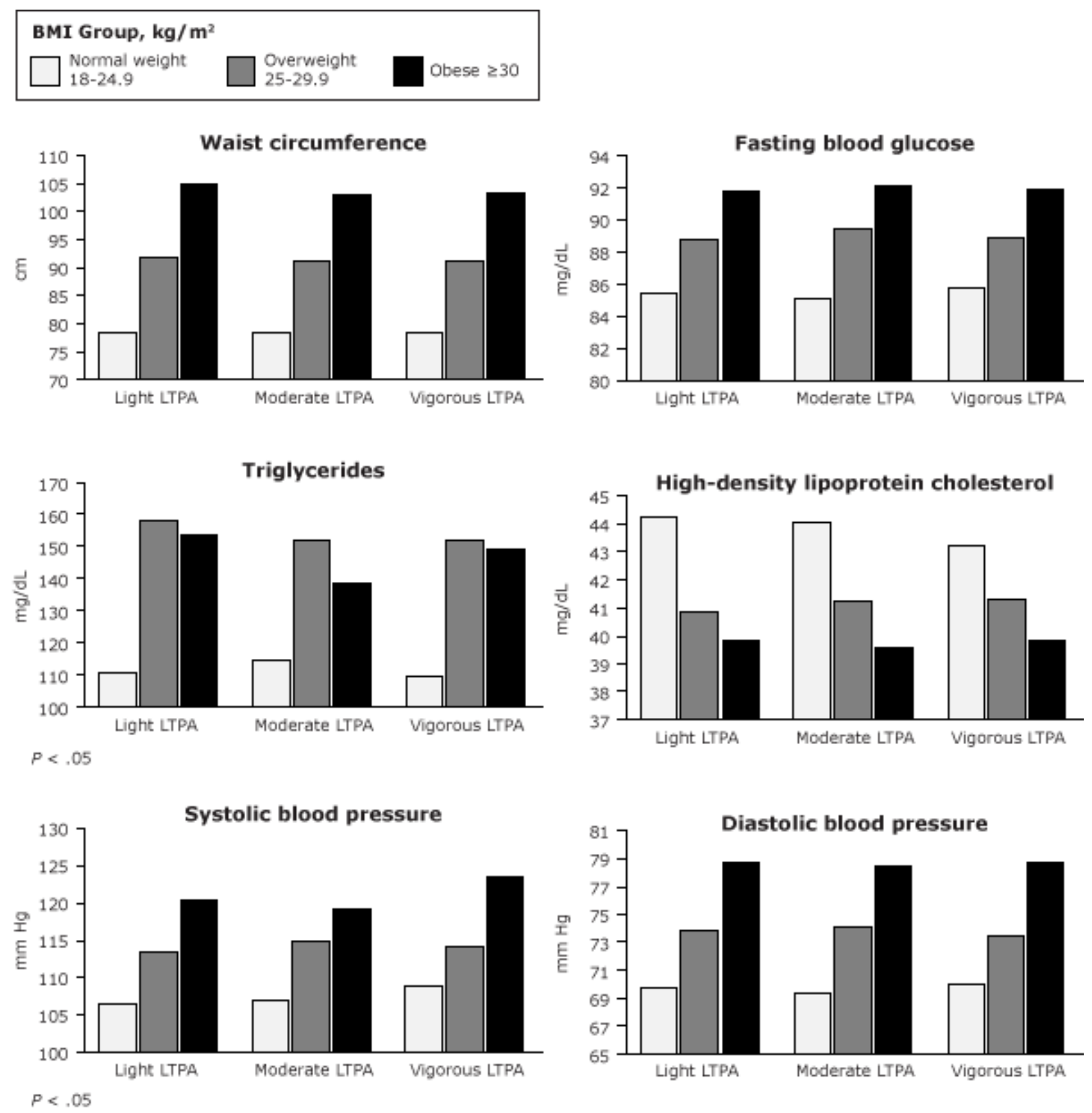

Figure. Systolic blood pressure and triglyceride levels differed significantly for obese adults depending on their LTPA level. Abbreviations: BMI, body mass index; LTPA, leisure-time physical activity. [A tabular version of this figure is also available.]

\section{Discussion}

Our results demonstrate that for the normal-weight group with a sedentary lifestyle (light LTPA), the risk of having higher triglyceride levels was $46 \%$ and lower HDL cholesterol was $15 \%$ higher than for those with vigorous LTPA. Overweight people with vigorous LTPA had lower fasting blood glucose than moderate LTPA groups. Overweight people with a sedentary lifestyle had a higher risk of MetS than those who participated in vigorous LTPA. Previous studies suggested that increasing levels of LTPA, in terms of duration or intensity, were linearly associated with reduced risk of MetS (12).

Despite these findings, the results of some studies show no association between physical activity and MetS (13). The lack of association could be related to the criteria used for MetS, age range, or limited number of participants. Like us, other researchers found that elevated levels of HDL cholesterol were associated with vigorous LTPA, and a sedentary lifestyle was positively associated with elevated levels of triglycerides and fasting blood glucose (14). Unexpectedly, the risk of having elevated systolic blood pressure in the normal-weight and obese groups with vigorous LTPA was nearly 
$60 \%$ higher than in the overweight group. The difference in systolic blood pressure (3 $\mathrm{mm} \mathrm{Hg}$ ) between LTPA groups has no clinical importance but could be caused by regular participation in moderate or vigorous physical activity (15).

Our study had a few limitations. First, this study was cross-sectional, so causality cannot be determined. Second, selfreported LTPA did not provide accurate estimates of absolute amounts of activity.

Engaging in any LTPA was associated with lower prevalence of MetS. Future studies are needed to investigate the association between various kinds of physical activity and MetS by considering factors that affect lifestyle, such as socioeconomic status.

\section{Acknowledgments}

We acknowledge Ms Nilufar Shiva for language editing of this manuscript and the staff and participants in the TLGS study for their cooperation. This work was funded and supported by the Research Institute for Endocrine Sciences of Shahid Beheshti University, Tehran, Iran.

\section{Author Information}

Corresponding Author: Farhad Hosseinpanah, Obesity Research Center, Research Institute for Endocrine Sciences, Shahid Beheshti University of Medical Sciences, PO Box 19395-476, Tehran, Iran. Telephone: +98-21-22432500. Email: fhospanah@endocrine.ac.ir.

Author Affiliations: Bita Faam, Atieh Amouzegar, Arash Ghanbarian, Golaleh Asghari, Fereidoun Azizi, Shahid Beheshti University of Medical Sciences, Tehran, Iran.

\section{References}

1. Eckel RH, Grundy SM, Zimmet PZ. The metabolic syndrome. Lancet 2005;365(9468):1415-28. CrossRef 洛 PubMed 逐

2. Zabetian A, Hadaegh F, Azizi F. Prevalence of metabolic syndrome in Iranian adult population, concordance between the IDF with the ATPIII and the WHO definitions. Diabetes Res Clin Pract 2007;77(2):251-7. CrossRef 通 PubMed 图

3. Grundy SM, Cleeman JI, Daniels SR, Donato KA, Eckel RH, Franklin BA, et al. Diagnosis and management of the metabolic syndrome: an American Heart Association/National Heart, Lung, and Blood Institute scientific statement: executive summary. Crit Pathw Cardiol 2005;4(4):198-203. PubMed 虑

4. Lakka TA, Laaksonen DE. Physical activity in prevention and treatment of the metabolic syndrome. Appl Physiol Nutr Metab 2007;32(1):76-88. CrossRef 密 PubMed 图

5. Azizi F, Rahmani M, Emami H, Mirmiran P, Hajipour R, Madjid M, et al. Cardiovascular risk factors in an Iranian urban population: Tehran Lipid and Glucose Study (phase 1). Soz Praventivmed 2002;47(6):408-26. CrossRef 卖 PubMed 图

6. Delavari A, Forouzanfar MH, Alikhani S, Sharifian A, Kelishadi R. First nationwide study of the prevalence of the metabolic syndrome and optimal cutoff points of waist circumference in the Middle East: the national survey of risk factors for noncommunicable diseases of Iran. Diabetes Care 2009;32(6):1092-7. CrossRef 图 PubMed 图

7. Alberti KG, Eckel RH, Grundy SM, Zimmet PZ, Cleeman JI, Donato KA, et al. Harmonizing the metabolic syndrome: a joint interim statement of the International Diabetes Federation Task Force on Epidemiology and Prevention; National Heart, Lung, and Blood Institute; American Heart Association; World Heart Federation; International Atherosclerosis Society; and International Association for the Study of Obesity. Circulation

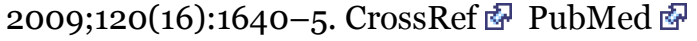

8. Kriska AM, Edelstein SL, Hamman RF, Otto A, Bray GA, Mayer-Davis EJ, et al. Physical activity in individuals at risk for diabetes: Diabetes Prevention Program. Med Sci Sports Exerc 2006;38(5):826-32. CrossRef 虚 PubMed 密

9. Momenan AA, Delshad M, Sarbazi N, Rezaei Ghaleh N, Ghanbarian A, Azizi F. Reliability and validity of the Modifiable Activity Questionnaire (MAQ) in an Iranian urban adult population. Arch Iran Med 2012;15(5):27982. PubMed 图

10. Sesso HD, Paffenbarger RS Jr, Lee IM. Physical activity and coronary heart disease in men. The Harvard Alumni Health Study. Circulation 2000;102(9):975-80.

11. Mirmiran P, Esfahani FH, Mehrabi Y, Hedayati M, Azizi F. Reliability and relative validity of an FFQ for nutrients in the Tehran Lipid and Glucose Study. Public Health Nutr 2010;13(5):654-62. CrossRef 處 PubMed 
Preventing Chronic Disease I Leisure-Time Physical Activity and Its Association With M... Page 5 of 8

12. Méndez-Hernández P, Flores Y, Siani C, Lamure M, Dosamantes-Carrasco LD, Halley-Castillo E. Physical activity and risk of metabolic syndrome in an urban Mexican cohort. BMC Public Health 2009;9:276. CrossRef 處 PubMed 这

13. Dalacorte RR, Reichert CL, Vieira JL. Metabolic syndrome and physical activity in southern Brazilian communitydwelling elders: a population-based, cross-sectional study. BMC Public Health 2009;9:25. CrossRef 图 PubMed 图

14. Li CL, Lin JD, Lee SJ, Tseng RF. Associations between the metabolic syndrome and its components, watching television and physical activity. Public Health 2007;121(2):83-91. CrossRef 总 PubMed 必

15. Pescatello LS, Guidry MA, Blanchard BE, Kerr A, Taylor AL, Johnson AN. Exercise intensity alters postexercise hypotension. J Hypertens 2004;22(10):1881-8. CrossRef 图 PubMed 图

\section{Tables}

Table 1. Demographic and Metabolic Characteristics of Participants by Body Mass Index (BMI)a, the Tehran Lipid and Glucose Study, 2005-2008b

\begin{tabular}{|c|c|c|c|c|}
\hline Variable & $\begin{array}{c}\text { BMI } 18-24.9 \mathrm{~kg} / \mathrm{m}^{2} \\
(\mathrm{n}=1,565)\end{array}$ & $\begin{array}{c}\text { BMI } 25-29.9 \mathrm{~kg} / \mathrm{m}^{2} \\
(\mathrm{n}=1,992)\end{array}$ & $\begin{array}{c}\mathrm{BMI} \geq 30 \mathrm{~kg} / \mathrm{m}^{2} \\
(\mathrm{n}=1,108)\end{array}$ & $\begin{array}{c}\text { Total }(n= \\
4,665)\end{array}$ \\
\hline Age, y (SD) & $34.8(13.6)$ & $42.7(13.1)$ & $45.3(12.6)$ & 40.7 (13.9) \\
\hline No. of men $(\%)$ & $716(45.5)$ & 915 (45.9) & $345(31.1)$ & $1,976(42.4)$ \\
\hline \multicolumn{5}{|l|}{ Smoker, n (\%) } \\
\hline Current & $172(11.1)$ & $226(11.4)$ & $99(8.9)$ & $497(10.6)$ \\
\hline Past & $112(7.2)$ & $174(8.7)$ & $67(6.1)$ & $353(7.6)$ \\
\hline \multicolumn{5}{|l|}{ Clinical measurement } \\
\hline $\begin{array}{l}\text { No. with metabolic syndrome } \\
(\%)\end{array}$ & $94(6.0)$ & $729(36.6)$ & $645(58.2)^{c}$ & $1,468(31.5)$ \\
\hline Weight, kg (SD) & $61.4(8.3)$ & $73.5(9.2)$ & $85.9(12.7)^{c}$ & $72.4(13.5)$ \\
\hline Height, cm (SD) & $165.2(9.5)$ & $163.6(9.6)$ & $160.3(9.8)$ & $163.3(9.8)$ \\
\hline Waist circumference, cm (SD) & $78.1(8.6)$ & $90.8(8.2)$ & $102.1(9.3)^{c}$ & $89.2(12.4)$ \\
\hline $\mathrm{BMI}, \mathrm{kg} / \mathrm{m}^{2}(\mathrm{SD})$ & $22.4(1.8)$ & $27.4(1.4)$ & $33.3(3.1)^{c}$ & $27.1(4.6)$ \\
\hline $\begin{array}{l}\text { Fasting blood glucose, } \mathrm{mg} / \mathrm{dL} \\
\text { (SD) }\end{array}$ & $85.2(1.1)$ & $88.9(1.1)$ & $91.2(1.1)^{\mathrm{c}}$ & $88.2(1.1)$ \\
\hline Triglycerides, mg/dL (SD) & $111.4(1.6)$ & $153.6(1.6)$ & $147.1(1.6)^{c}$ & $143.7(88.7)$ \\
\hline $\begin{array}{l}\text { High-density lipoprotein } \\
\text { cholesterol, mg/dL (SD) }\end{array}$ & $44.1(10.5)$ & $41.3(9.7)$ & $41.2(9.9)^{c}$ & $42.2(10.1)$ \\
\hline $\begin{array}{l}\text { Systolic blood pressure, } \mathrm{mm} \mathrm{Hg} \\
\text { (SD) }\end{array}$ & $106.9(14.3)$ & $113.8(16.3)$ & $119.1(17.2)^{c}$ & $112.7(16.6)$ \\
\hline $\begin{array}{l}\text { Diastolic blood pressure, } \mathrm{mm} \\
\mathrm{Hg},(\mathrm{SD})\end{array}$ & $69.5(8.9)$ & $73.7(9.6)$ & $77.7(9.9)^{c}$ & $73.2(10.1)$ \\
\hline \multicolumn{5}{|l|}{ Education (\%) } \\
\hline Elementary or secondary & $256(16.4)$ & 596 (29.9) & $486(43.9)^{c}$ & $1,338(28.7)$ \\
\hline High school & $827(52.8)$ & $875(43.9)$ & $423(38.2)^{c}$ & $2,125(45.6)$ \\
\hline Bachelor's degree or higher & $452(28.9)$ & $458(23.0)$ & $145(13.1)^{c}$ & $1,055(22.6)$ \\
\hline \multicolumn{5}{|c|}{ Leisure-time physical activity (LTPA), n (\%) } \\
\hline Light LTPA (MET <600 min/wk) & $794(50.7)$ & $1,011(50.8)$ & $578(52.2)$ & $2,383(51.1)$ \\
\hline $\begin{array}{l}\text { Moderate LTPA (MET 600-1,499 } \\
\mathrm{min} / \mathrm{wk} \text { ) }\end{array}$ & $488(31.2)$ & $637(32.0)$ & 365 (32.9) & $1,490(31.9)$ \\
\hline
\end{tabular}


Preventing Chronic Disease I Leisure-Time Physical Activity and Its Association With M... Page 6 of 8

\begin{tabular}{|c|c|c|c|c|}
\hline Variable & $\begin{array}{c}\text { BMI } 18-24.9 \mathrm{~kg} / \mathrm{m}^{2} \\
(\mathrm{n}=1,565)\end{array}$ & $\begin{array}{c}\text { BMI } 25-29.9 \mathrm{~kg} / \mathrm{m}^{2} \\
(\mathrm{n}=1,992)\end{array}$ & $\begin{array}{c}\text { BMI } \geq 30 \mathrm{~kg} / \mathrm{m}^{2} \\
(\mathrm{n}=1,108)\end{array}$ & $\begin{array}{c}\text { Total }(n= \\
4,665)\end{array}$ \\
\hline $\begin{array}{l}\text { Vigorous LTPA (MET } \geq 1,500 \\
\mathrm{~min} / \mathrm{wk} \text { ) }\end{array}$ & $283(18.1)$ & $344(17.3)$ & $165(14.9)$ & $792(17.0)$ \\
\hline
\end{tabular}

Abbreviations: SD, standard deviation; MET, metabolic equivalent task.

a BMI is calculated as weight in kilograms divided by the square of height in meters $\left(\mathrm{kg} / \mathrm{m}^{2}\right)$.

b Data were log transformed. Values for all of the variables were adjusted for age and sex.

c Differences between groups were assessed by using analysis of covariance; significance was set at $P<.05$.

Table 2. Association Between Leisure-Time Physical Activitya and Metabolic Return' Syndrome Components, by Body Mass Index (BMI)b, Tehran Lipid and

Glucose Study, 2005-2008c

\begin{tabular}{|c|c|c|c|c|}
\hline $\begin{array}{l}\text { Metabolic Syndrome } \\
\text { Componment }\end{array}$ & $\begin{array}{l}\text { Crude Odds Ratio (95\% } \\
\text { CI) }\end{array}$ & $\begin{array}{c}P \\
\text { Value }\end{array}$ & $\begin{array}{c}\text { Adjusted Odds Ratio (95\% } \\
\text { CI) }\end{array}$ & $\begin{array}{c}P \\
\text { Value }\end{array}$ \\
\hline \multicolumn{5}{|c|}{ BMI $18-24.9 \mathrm{~kg} / \mathrm{m}^{2}$} \\
\hline \multicolumn{5}{|l|}{ Abdominal obesity } \\
\hline Vigorous LTPA & \multicolumn{4}{|c|}{1 [Reference] } \\
\hline Moderate LTPA & $1.29(0.47-3.50)$ & .62 & $0.25(0.03-2.11)$ & .21 \\
\hline Light LTPA & $1.76(0.63-4.90)$ & .28 & $1.42(0.22-9.38)$ & .71 \\
\hline \multicolumn{5}{|l|}{ Fasting blood glucose } \\
\hline Vigorous LTPA & \multicolumn{4}{|c|}{1 [Reference] } \\
\hline Moderate LTPA & $1.02(0.45-1.57)$ & .60 & $1.31(0.14-2.31)$ & .81 \\
\hline Light LTPA & $1.05(0.52-1.93)$ & .98 & $1.52(0.19-2.51)$ & .61 \\
\hline \multicolumn{5}{|l|}{ Elevated triglycerides } \\
\hline Vigorous LTPA & \multicolumn{4}{|c|}{1 [Reference] } \\
\hline Moderate LTPA & $1.12(0.78-1.61)$ & .53 & $0.79(0.37-1.68)$ & .54 \\
\hline Light LTPA & $1.46(1.01-2.14)$ & .049 & $1.01(0.46-2.21)$ & .98 \\
\hline \multicolumn{5}{|l|}{ Low HDL-C } \\
\hline Vigorous LTPA & \multicolumn{4}{|c|}{1 [Reference] } \\
\hline Moderate LTPA & $0.76(0.58-1.01)$ & .06 & $1.00(0.55-1.74)$ & .95 \\
\hline Light LTPA & $1.15(1.05-2.33)$ & .03 & $0.77(0.42-1.41$ & .41 \\
\hline \multicolumn{5}{|l|}{ Elevated SBP } \\
\hline Vigorous LTPA & \multicolumn{4}{|c|}{1 [Reference] } \\
\hline Moderate LTPA & $0.52(0.31-0.86)$ & .01 & $1.02(0.24-4.31)$ & .97 \\
\hline Light LTPA & $0.69(0.40-1.17)$ & .17 & $1.05(0.22-4.48)$ & .99 \\
\hline \multicolumn{5}{|l|}{ Elevated DBP } \\
\hline Vigorous LTPA & \multicolumn{4}{|c|}{1 [Reference] } \\
\hline Moderate LTPA & $1.29(0.63-2.65)$ & .48 & $1.76(0.46-6.81)$ & .41 \\
\hline Light LTPA & $1.29(0.61-2.76)$ & .51 & $0.86(0.19-3.92)$ & .85 \\
\hline \multicolumn{5}{|l|}{ MetS } \\
\hline Vigorous LTPA & \multicolumn{4}{|c|}{1 [Reference] } \\
\hline Moderate LTPA & $0.98(0.51-1.59)$ & .73 & $1.41(0.28-7.13)$ & .41 \\
\hline
\end{tabular}


Preventing Chronic Disease I Leisure-Time Physical Activity and Its Association With M... Page 7 of 8

\begin{tabular}{|c|c|c|c|c|}
\hline $\begin{array}{l}\text { Metabolic Syndrome } \\
\text { Componment }\end{array}$ & $\begin{array}{c}\text { Crude Odds Ratio ( } 95 \% \\
\text { CI) }\end{array}$ & $\begin{array}{c}P \\
\text { Value }\end{array}$ & $\begin{array}{l}\text { Adjusted Odds Ratio (95\% } \\
\text { CI) }\end{array}$ & $\begin{array}{c}P \\
\text { Value }\end{array}$ \\
\hline Light LTPA & $0.96(0.52-1.76)$ & .91 & $1.12(0.19-6.37)$ & .12 \\
\hline \multicolumn{5}{|c|}{ BMI $25-29.9 \mathrm{~kg} / \mathrm{m}^{2}$} \\
\hline \multicolumn{5}{|l|}{ Abdominal obesity } \\
\hline Vigorous LTPA & \multicolumn{4}{|c|}{1 [Reference] } \\
\hline Moderate LTPA & $1.01(0.66-1.10)$ & .23 & $1.01(0.51-1.88)$ & .94 \\
\hline Light LTPA & $1.03(0.71-1.22)$ & .61 & $1.02(0.45-1.76)$ & .73 \\
\hline \multicolumn{5}{|l|}{ Fasting blood glucose } \\
\hline Vigorous LTPA & \multicolumn{4}{|c|}{1 [Reference] } \\
\hline Moderate LTPA & $0.80(0.54-1.19)$ & .27 & $1.65(1.37-3.23)$ & .02 \\
\hline Light LTPA & $1.01(0.67-1.53)$ & .95 & $1.47(1.28-3.31)$ & .06 \\
\hline \multicolumn{5}{|l|}{ Elevated triglycerides } \\
\hline Vigorous LTPA & \multicolumn{4}{|c|}{1 [Reference] } \\
\hline Moderate LTPA & $1.09(0.85-1.41)$ & .47 & $1.06(0.61-1.87)$ & .82 \\
\hline Light LTPA & $1.01(0.67-1.53)$ & .35 & $1.29(0.72-2.34)$ & .39 \\
\hline \multicolumn{5}{|l|}{ Low HDL-C } \\
\hline Vigorous LTPA & \multicolumn{4}{|c|}{1 [Reference] } \\
\hline Moderate LTPA & $1.03(0.75-1.23)$ & .75 & $1.24(0.72-2.15)$ & .43 \\
\hline Light LTPA & $1.04(0.74-1.25)$ & .78 & $1.35(0.76-2.41)$ & .31 \\
\hline \multicolumn{5}{|l|}{ Elevated SBP } \\
\hline Vigorous LTPA & \multicolumn{4}{|c|}{1 [Reference] } \\
\hline Moderate LTPA & $0.78(0.56-1.08)$ & .14 & $0.74(0.33-1.65)$ & .46 \\
\hline Light LTPA & $1.00(0.70-1.41)$ & .98 & $1.22(0.54-2.77)$ & .64 \\
\hline \multicolumn{5}{|l|}{ Elevated DBP } \\
\hline Vigorous LTPA & \multicolumn{4}{|c|}{1 [Reference] } \\
\hline Moderate LTPA & $1.00(0.56-1.16)$ & .25 & $1.23(0.53-2.84)$ & .63 \\
\hline Light LTPA & $1.01(0.65-1.41)$ & .82 & $1.17(0.49-2.81)$ & .72 \\
\hline \multicolumn{5}{|l|}{ MetS } \\
\hline Vigorous LTPA & \multicolumn{4}{|c|}{1 [Reference] } \\
\hline Moderate LTPA & $0.93(0.72-1.20)$ & .59 & $1.87(0.95-3.67)$ & .07 \\
\hline Light LTPA & $1.07(0.81-1.40)$ & .63 & $2.08(1.03-4.21)$ & .04 \\
\hline \multicolumn{5}{|c|}{$\mathrm{BMI} \geq 30 \mathrm{~km} / \mathrm{m}^{2}$} \\
\hline \multicolumn{5}{|l|}{ Abdominal obesity } \\
\hline Vigorous LTPA & \multicolumn{4}{|c|}{1 [Reference] } \\
\hline Moderate LTPA & $1.42(0.92-2.16)$ & .11 & $1.12(0.35-3.59)$ & .85 \\
\hline Light LTPA & $0.88(0.57-1.36)$ & .58 & $0.75(0.23-2.42)$ & .63 \\
\hline \multicolumn{5}{|l|}{ Fasting blood glucose } \\
\hline Vigorous LTPA & \multicolumn{4}{|c|}{1 [Reference] } \\
\hline Moderate LTPA & $0.69(0.46-1.07)$ & .09 & $0.47(0.18-1.22)$ & .12 \\
\hline Light LTPA & $0.76(0.49-1.98)$ & .24 & $0.79(0.31-2.08)$ & .63 \\
\hline
\end{tabular}


Preventing Chronic Disease I Leisure-Time Physical Activity and Its Association With M... Page 8 of 8

\begin{tabular}{|c|c|c|c|c|}
\hline $\begin{array}{l}\text { Metabolic Syndrome } \\
\text { Componment }\end{array}$ & $\begin{array}{l}\text { Crude Odds Ratio (95\% } \\
\text { CI) }\end{array}$ & $\begin{array}{c}P \\
\text { Value }\end{array}$ & $\begin{array}{c}\text { Adjusted Odds Ratio (95\% } \\
\text { CI) }\end{array}$ & $\begin{array}{c}P \\
\text { Value }\end{array}$ \\
\hline \multicolumn{5}{|l|}{ Elevated triglycerides } \\
\hline Vigorous LTPA & \multicolumn{4}{|c|}{1 [Reference] } \\
\hline Moderate LTPA & $1.12(0.79-1.58)$ & .52 & $1.85(0.81-4.22)$ & .14 \\
\hline Light LTPA & $0.80(0.55-1.16)$ & .24 & $1.39(0.58-3.31)$ & .46 \\
\hline \multicolumn{5}{|l|}{ Low HDL-C } \\
\hline Vigorous LTPA & \multicolumn{4}{|c|}{1 [Reference] } \\
\hline Moderate LTPA & $0.77(0.54-0.89)$ & .15 & $1.13(0.51-2.51)$ & .77 \\
\hline Light LTPA & $1.08(0.63-1.19)$ & .68 & $1.57(0.68-3.63)$ & .29 \\
\hline \multicolumn{5}{|l|}{ Elevated SBP } \\
\hline Vigorous LTPA & \multicolumn{4}{|c|}{1 [Reference] } \\
\hline Moderate LTPA & $0.58(0.39-0.64)$ & .005 & $0.52(0.21-1.33)$ & .17 \\
\hline Light LTPA & $0.60(0.41-0.91)$ & .01 & $0.55(0.21-1.46)$ & .23 \\
\hline \multicolumn{5}{|l|}{ Elevated DBP } \\
\hline Vigorous LTPA & \multicolumn{4}{|c|}{1 [Reference] } \\
\hline Moderate LTPA & $0.79(0.54-1.18)$ & .26 & $0.67(0.28-1.58)$ & .36 \\
\hline Light LTPA & $0.65(0.43-1.00)$ & .053 & $0.41(0.16-1.02)$ & .06 \\
\hline \multicolumn{5}{|l|}{ MetS } \\
\hline Vigorous LTPA & \multicolumn{4}{|c|}{1 [Reference] } \\
\hline Moderate LTPA & $0.92(0.65-1.32)$ & .68 & $1.01(0.43-2.21)$ & .96 \\
\hline Light LTPA & $0.72(0.49-1.04)$ & .08 & $1.03(0.38-2.11)$ & .82 \\
\hline
\end{tabular}

Abbreviations: CI, confidence interval; LTPA, leisure time physical activity; HDL-C, high density lipoprotein cholesterol; SBP, systolic blood pressure; DBP; diastolic blood pressure; MetS, metabolic syndrome.

a Light LTPA is metabolic Equivalent of Task (MET) <600 min/wk; moderate LTPA is MET 600-1,499 min/wk; and vigorous LTPA is MET $\geq 1,500 \mathrm{~min} / \mathrm{wk}$.

b BMI is calculated as weight in kilograms divided by the square of height in meters $\left(\mathrm{kg} / \mathrm{m}^{2}\right)$.

c Values were adjusted for sex, age, smoking status, education levels, and calorie intake.

The opinions expressed by authors contributing to this journal do not necessarily reflect the opinions of the U.S. Department of Health and Human Services, the Public Health Service, the Centers for Disease Control and Prevention, or the authors' affiliated institutions.

The RIS file format is a text file containing bibliographic citations. These files are best suited for import into

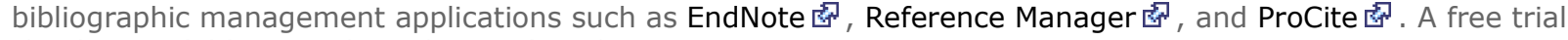
download is available at each application's web site.

For Questions About This Article Contact pcdeditor@cdc.gov

Page last reviewed: March 14, 2013

Page last updated: March 14, 2013

Content source: National Center for Chronic Disease Prevention and Health Promotion

Centers for Disease Control and Prevention 1600 Clifton Rd. Atlanta, GA 30333, USA

80o-CDC-INFO (80o-232-4636) TTY: (888) 232-6348 - Contact CDC-INFO 